\title{
Yeast beta-Glucan effects on gut microbiota for regulating insulin signaling system on alzheimer's disease
}

\author{
Xu Mengdai, Mo Xiaoxing, Huang Hao, Liu Liegang and Yang Wei \\ Tongji Medical College, Huazhong University of Science and Technology, Wuhan, China
}

\begin{abstract}
Introduction: Alzheimer's disease (AD) is a progressive neurodegenerative disease for dementia in adults. The main pathological alterations are extracellular senile plaque deposits, intracellular neurofibrillary tangles and neuronal apoptosis. Recent researches indicated that T2D is closely with AD by insulin resistance in central nerves system progression. Nevertheless, the pathological mechanism remains unclear and treatment is limited. $\beta$-glucan extracted from yeast, as a dietary fiber with high bioactivity, edible, good taste and easy-obtainable, have been showed abilities such as anti-diabetic, anti-inflammatory and prebiotic. Based on this, $\beta$-glucan can reduce insulin resistance and maintain gut microbiota thereby alleviating lesions of early AD.
\end{abstract}

Materials and Methods: In this study, we used 36 male wild-type C57BL/6J mice were divided to 3 groups (control (C) mice injected i. c.v. with $0.9 \%$ saline as a vehicle, mice injected i.c.v. with $\mathrm{A} \beta_{1-42}\left(\mathrm{~A} \beta_{1-42}\right.$ group), mice injected with $\mathrm{A} \beta_{1-42}$ and soluble yeast $\beta$-glucan $100 \mathrm{mg} / \mathrm{kg}$ body weight by oral gavage daily (o.g.) for $\left.5 \mathrm{wks}\left(\mathrm{A} \beta_{1-42}+\mathrm{Glu}\right)\right)$. H\&E method was detected for structure of hippocampus. Morris water maze test was performed to assess the cognitive performance of A $\beta$-infusion mice. The microbiota composition was analyzed by 16sRNA sequencing. The levels of inflammatory were measured in hippocampal and plasma by Meso-scale Discovery (MSD). Western blot was performed to detect the level of protein in insulin signaling pathway. One-way ANNOVA with Student-Newman-Keuls was applied for the data analysis through SPSS software version 22.0.

Results: As demonstrated by H\&E sections, $\beta$-glucan reduced neuron damage in AD mice hippocampus. Decreased the levels of A $\beta$ and phosphorylation of Tau protein expression in hippocampus $(\mathrm{P}<0.05)$ and ameliorated insulin resistance $(\mathrm{p}$-IRS-1) in hippocampus $(\mathrm{P}<0.05)$. According by results from MSD and Western-blot that showed TNF- $\alpha(\mathrm{P}<0.05)$, phosphorylated JNK $(\mathrm{P}<0.01)$ and Tau were up-regulated in AD but $\beta$-glucan group decreased. In addition, the abundance of beneficial bacteria in $\beta$-glucan mice is increased (g_Alistipes, g_Rikeenella and g_Saccharibecteria genera incerae sedies).

Discussion: Summary, this study illustrated that $\beta$-glucan regulated insulin signaling for ameliorating learning and memory deficit in AD. Due to $\beta$-glucan can not pass Blood-Brain-Barrier, we hypothesized that $\beta$-glucan could regulates gut microbiota by metabolites for ameliorating neuron damage. Our study provides new ideas for the prevention of AD.

\section{Conflict of Interest}

There is no conflict of interest. 